\title{
Inference Engine in an Intelligent Ship Course-Keeping System
}

\author{
Piotr Borkowski \\ Maritime University of Szczecin, Wały Chrobrego 1, 70500 Szczecin, Poland \\ Correspondence should be addressed to Piotr Borkowski; p.borkowski@am.szczecin.pl \\ Received 5 September 2017; Revised 16 October 2017; Accepted 9 November 2017; Published 29 November 2017 \\ Academic Editor: Thomas DeMarse \\ Copyright (C) 2017 Piotr Borkowski. This is an open access article distributed under the Creative Commons Attribution License, \\ which permits unrestricted use, distribution, and reproduction in any medium, provided the original work is properly cited.

\begin{abstract}
The article presents an original design of an expert system, whose function is to automatically stabilize ship's course. The focus is put on the inference engine, a mechanism that consists of two functional components. One is responsible for the construction of state space regions, implemented on the basis of properly processed signals recorded by sensors from the input and output of an object. The other component is responsible for generating a control decision based on the knowledge obtained in the first module. The computing experiments described herein prove the effective and correct operation of the proposed system.
\end{abstract}

\section{Introduction}

The importance of maritime transport in the global economy is expressed primarily by the quantity of cargoes carried by sea, currently estimated at $80 \%$. If we assume the transport performance as a criterion, the share of maritime transport in global trade exceeds $90 \%$ [1]. The actual competitiveness of maritime transport in relation to other modes results in increasingly larger seaborne trade. Consequently, traffic intensity, tonnage, and speeds of vessels also increase. This in turn affects the level of safety of people, vessels, cargo, and the environment. In order to improve safety and increase the efficiency and competitiveness of marine transport services, more and more advanced navigational systems and equipment are installed on board ships and in land-based vessel traffic control centers. The benefits are as follows:

(i) Material benefits associated with the reduction of losses and damage to and sinking of vessels

(ii) Economic benefits due to lower operating costs and shorter voyage

(iii) Environmental protection and prevention of environmental disasters caused by collisions of vessels carrying dangerous goods

One of the typical tasks related to the safety of navigation is the problem of automatic course stabilization, consisting in automatic maintenance of the ship on a preset course under conditions of external disturbances, as well as bringing the ship automatically back on a preset course after its previous alteration. The ship's course-keeping capability is crucial, because nonoptimal control of the rudder involves the loss of average speed, covering a longer track, lengthened time of the voyage, and more fuel consumption, which in consequence raises total operating costs. The associated problem is steering gear overload that may lead to a critical breakdown. Most importantly, uncontrollable yawing, especially on waterways with increased traffic, affects the level of safety, increasing a risk of collision [2].

The history of automatic ship movement control devices dated back to the early 20th century. The invention of gyrocompass (1908, H. Anschutz), a device capable of indicating north with high accuracy, also on ships made of steel (unlike the compass), enabled the development of autopilots (1911, E. Sperry). Initially, such devices were simple mechanisms, transforming proportionally a signal from the gyrocompass into a control decision, so their designs were mostly based on the concept of the $\mathrm{P}$ regulator. From this period the publications $[3,4]$ are worth noting, regarded as pioneer works in the field.

The helmsman's actions on the bridge are similar to the operation of PID controller, that is, it proportionally accounts for deviation from ship's course (term P: a proportional component), action of factors causing permanent deviation from the course (term I: an integral component), and speed of deviating from the course (term $\mathrm{D}$ : a derivative component). 


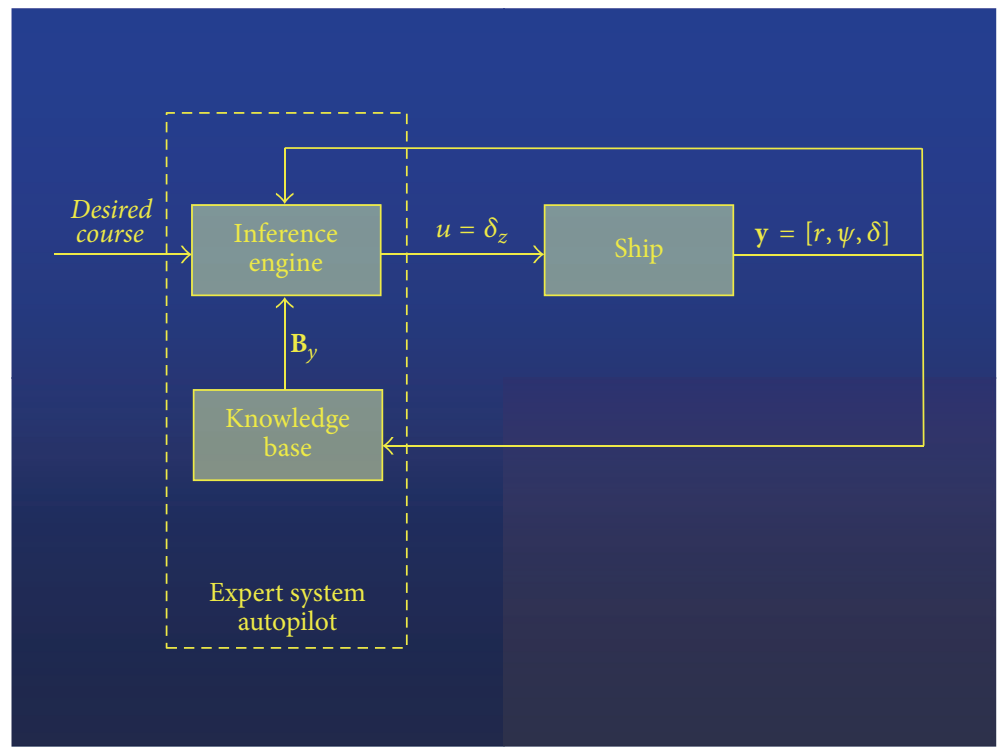

FIGURE 1: The circulation of signals in the proposed expert system.

It is therefore natural that designs of subsequent autopilots usually were based on the PID controller concept, sometimes on PD controllers. Till the 1990s, the PID control method was a dominant solution, and specific examples can be found in the works [5-7].

Because dynamic properties of a ship are not constant (they depend on vessel type and sailing conditions), the $\mathrm{PID} / \mathrm{PD}$ controller setpoints should also change, especially if the autopilot is to serve for maintaining the ship on course, as well as for course alteration to a new value. This requirement has initiated a whole range of new concepts based on the notion of space states and stochastic control theory. These solutions often make use of Kalman filter or reference model methods. Examples of adaptive autopilot designs can be found in works [8-15].

The simplest adaptive autopilots based on the PID (PD) controller concept have higher quality of control than previous solutions. However, their idea is based mainly on the linear model of object dynamics. Developments of the control theory included attempts to design autopilots operating on the basis of nonlinear control techniques. Some examples can be found in the publications [16-25].

Another solution bypassing the problem of nonlinearity is the use of computer technologies and artificial intelligence methods. The number of publications on this subject is constantly on the rise. Example references are [26-36]. The system herein described fits into that area of research. It is not a classic expert system, where expert knowledge is recorded in the form of rules. In the proposed solution, the knowledge is represented by properly processed signals recorded by sensors from the object input and output. The inference engine is crucial for the proposed expert system of ship's course stabilization. It consists of two functional components or modules. One of them is responsible for the construction of space states, a basis for the other module to generate a control decision.
In this original method, it is possible to overcome difficulties that occur in developing typical control algorithms for a complex and nonlinear model affected by strong external disturbances (wind, waves), while providing high quality control.

\section{The Design of an Intelligent System of Ship Course Stabilization}

The circulation of signals in the proposed expert system of ship course stabilization (course autopilot) is shown in Figure 1. In the inference engine, for a given object output $\mathbf{y}=[r, \psi, \delta]$ ( $r$ : angular velocity, $\psi$ : deviation from course, and $\delta$ : rudder angle), a control decision $u$ is generated, that is, the preset rudder angle $\delta_{z}$, on the basis of quantities retrieved from the knowledge base $\mathbf{B}_{y}$. It is so determined as to stabilize the defined quantity, that is, ship's course. This process is repeated at time unit intervals $\Delta t$.

Let the symmetric, closed interval $\left\langle r_{\min }, r_{\max }\right\rangle$ be a set of all angular velocities that the object may assume. After discretization, we obtain this set:

$$
\begin{aligned}
& \left\{r_{\min }, r_{\min }+\Delta r, r_{\min }+2 \Delta r, \ldots, r_{\min }+\left(n_{r}-1\right) \Delta r\right. \\
& \left.\quad=r_{\max }\right\},
\end{aligned}
$$

where

$$
\begin{aligned}
& \Delta r \text { is discretization unit, } \\
& n_{r} \text { is number of elements in the set (1). }
\end{aligned}
$$

Similarly, as a result of discretization of possible values of rudder angle (i.e., the interval $\left\langle\delta_{\min }, \delta_{\max }\right\rangle$ ), we get a set:

$$
\begin{aligned}
& \left\{\delta_{\min }, \delta_{\min }+\Delta \delta, \delta_{\min }+2 \Delta \delta, \ldots, \delta_{\min }+\left(n_{\delta}-1\right) \Delta \delta\right. \\
& \left.\quad=\delta_{\max }\right\}
\end{aligned}
$$


where

\section{$\Delta \delta$ is discretization unit,}

$n_{\delta}$ is number of elements in the set (2).

The knowledge base contains object output signals recorded in an ordered manner (resp., angular velocity, deviation from the course, and rudder angle), depending on the discretized state of the object and discretized control decisions. The output signal is here to be understood as measurement of object state after one time unit $\Delta t$. For practical reasons, the signals may be replaced by data obtained from any complex hydrodynamic model describing the object and the impact of the environment. However, to avoid a system with an open loop, a hydrodynamic model has to be adapted by a parametric or structural method to changing conditions, on the basis of real signals recorded from the object output. An ordered record of signals may be implemented in the form of multidimensional arrays with these values:

$$
\begin{gathered}
r[i, j, k] ; \\
\psi[i, j, k] ; \\
\delta[i, j] \\
\left(0 \leq i<n_{\delta} ; 0 \leq j<n_{\delta} ; 0 \leq k<n_{r}\right),
\end{gathered}
$$

where

$r[i, j, k]$ is value of object angular velocity after one time unit $\Delta t$; when at a current instant the angular velocity of the object is $r_{\min }+k \Delta r$, rudder angle is $\delta_{\min }+j \Delta \delta$, and the control decision has the form $\delta_{\min }+i \Delta \delta$,

$\psi[i, j, k]$ is value of object course deviation after one time unit $\Delta t$; when at a current instant the object angular velocity is $r_{\min }+k \Delta r$, rudder angle is $\delta_{\min }+$ $j \Delta \delta$, course deviation is equal to zero, and the control decision has the form $\delta_{\min }+i \Delta \delta$,

$\delta[i, j]$ is value of rudder angle after one time unit $\Delta t$; when at a current instant the rudder angle is $\delta_{\min }+$ $j \Delta \delta$, and the control decision has the form $\delta_{\min }+i \Delta \delta$.

Array values (3) and linear interpolation used allow determining an object output for any initial state and any control decision in the form as elements of the set (2).

The following sections describe the inference engine of the proposed expert system, the crucial component in generating a control decision. The last section provides a proof of control system stability.

2.1. The Construction of State Space Regions. The first of two functionally important components of the inference engine of the proposed expert system is responsible for the construction of state space regions. A proper classification of state space regions will be a basis for the control algorithm described in the next section.

Let the closed zero region $\mathrm{O}_{0}$ be a set of object states in the form:

$$
O_{0}=\left\{(r, \psi, \delta):|r| \leq r_{0} \wedge|\psi| \leq \psi_{0} \wedge|\delta| \leq \delta_{0}\right\},
$$

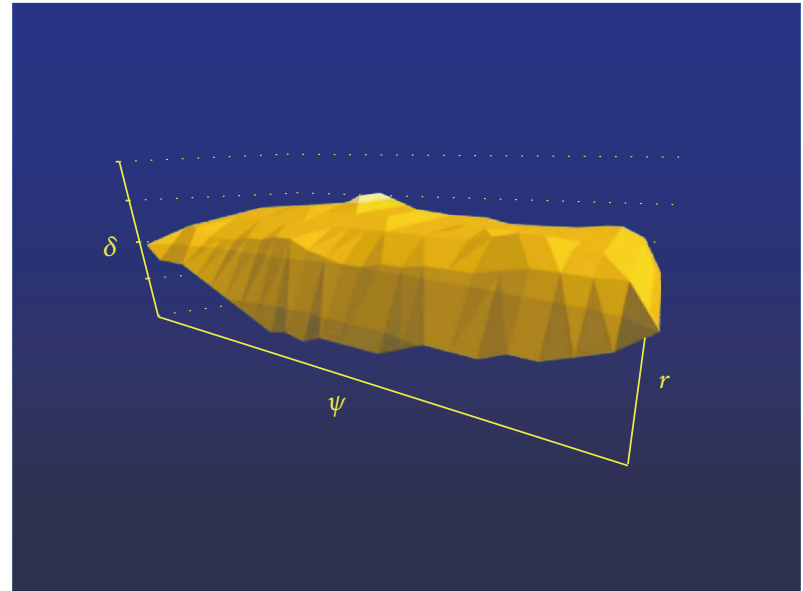

FIgURE 2: Visualization $O_{k}$.

where

$r_{0}, \psi_{0}, \delta_{0}$ is close to zero, positive values determined arbitrarily $\left(r_{0} \in(1), \delta_{0} \in(2)\right)$.

The zero region $\mathrm{O}_{0}$ is therefore a cuboid with the boundary. Parameters $r_{0}, \psi_{0}$, and $\delta_{0}$ should be arbitrarily selected so that their values are close to zero. They are thus established because the problem of ship course stabilization consists in bringing simultaneously the values of the angular velocity, course deviation, and rudder angle close to zero.

The closed $k$ th region $O_{k}$ (Figure 2) where $k \in N_{+}$is a set of object states for which there exists a sequence of control decisions not longer than $k$, generated at time unit intervals, bringing the object state to zero region $\mathrm{O}_{0}$.

The first region $O_{1}$ will be a set of such object states for which there exists a control decision that when executed results in the object state after one time unit $\Delta t$ getting to the zero region $\mathrm{O}_{0}$. The second region $\mathrm{O}_{2}$ is the sum of the first region $O_{1}$ and a set of object states for which there exists a control decision, leading to the state transition to the first region $O_{1}$ after one time unit $\Delta t$. In the same way, further regions are determined. It should be noted that the so-defined process of determining regions of state spaces is finite, because a set of all possible states is a cuboid with the boundary (angular velocity, deviation from the course, or rudder angle may only take values from a certain range). So there is some $k_{\max } \in N_{+}$, such that the region with this index will be a set of all possible states of the object (space of states):

$$
O_{k_{\max }}=\left\langle r_{\min }, r_{\max }\right\rangle \times\langle-\pi, \pi\rangle \times\left\langle\delta_{\min }, \delta_{\max }\right\rangle .
$$

The boundaries of state space regions are determined ascendingly starting from the first region $O_{1}$, to the region $O_{k}$. It can be noticed that for preset angular velocity and rudder angle, based on the values from the arrays (3), we can determine the ranges of course deviations for which there exist a control decision that after execution will result, after one time unit $\Delta t$, in the object state being moved to the region with an index lower by one than the region under consideration. With that relationship used iteratively, we get 
the boundaries of state space regions determined in a discrete manner. They can be written as two multidimensional arrays with the values:

$$
\begin{aligned}
& o \min [i, j, k] \\
& o \max [i, j, k] \\
&\left(0 \leq i<n_{r} ; 0 \leq j<n_{\delta} ; 0 \leq k<k_{\max }\right),
\end{aligned}
$$

where

$o \min [i, j, k]$ is value of the lower range limit of course deviation of the $k$ th region $O_{k}$ for object angular velocity $r_{\min }+i \Delta r$ and rudder angle $\delta_{\min }+j \Delta \delta$,

$o \max [i, j, k]$ is value of the upper range limit of course deviation of the $k$ th region $O_{k}$ for object angular velocity $r_{\min }+i \Delta r$ and rudder angle $\delta_{\min }+j \Delta \delta$.

The said course deviation range is to be understood as

$\emptyset \quad$ while $o \min [i, j, k]=o \max [i, j, k]$,

$$
\begin{aligned}
& \langle o \min [i, j, k], o \max [i, j, k]\rangle \\
& \text { while } o \min [i, j, k]<o \max [i, j, k], \\
& \langle-\pi, o \max [i, j, k]\rangle \cup\langle o \min [i, j, k], \pi\rangle \\
& \text { while } o \min [i, j, k]>o \max [i, j, k] .
\end{aligned}
$$

The determination of state space region boundaries (6) should take place for each change in the array values (3). Such procedure assures the adaptation of the system under consideration to changing conditions of ship movement.

2.2. The Control Algorithm. For the object output $\mathbf{y}=$ $[r(t), \psi(t), \delta(t)]$ at instant $t$, object states after a time unit $[r(t+\Delta t), \psi(t+\Delta t), \delta(t+\Delta t)]$ are determined by means of elements retrieved from the knowledge base $\left(\mathbf{B}_{y}: r[0\right.$ : $\left.n_{\delta}-1, j: j+1, k: k+1\right], \psi\left[0: n_{\delta}-1, j: j+1, k: k+1\right]$, $\delta\left[0: n_{\delta}-1, j: j+1\right]$, where $r_{\min }+k \Delta r \leq r(t)<r_{\min }+(k+1) \Delta r$, $\left.\delta_{\min }+j \Delta \delta \leq \delta(t)<\delta_{\min }+(j+1) \Delta \delta\right)$, depending on the discrete control decisions. It should be noted that for sufficiently small time increments $\Delta t$ the system will respond differently for some, not all, control decisions. This will limit the set of further analyzed cases.

The next step, made on the basis of array values (6), and with use of linear interpolation, defines the minimum indexes of regions where the object states will be found in the first step, depending on the analyzed control decisions (those, for which the system response was different after one time unit $\Delta t)$.

The generated control decision should correspond exactly with the smallest index determined in the previous step. However, this condition may prove to be inadequate, because it will be met by more than one of the analyzed control decisions. Let the set $\Delta_{z}$ mean all such control decisions. In this case, the most appropriate seems to be the decision that fulfils the condition:

$$
\delta_{z}=\min _{\delta \in \Delta_{z}}\{|\delta(t)-\delta|\}
$$

Condition (8), on the one hand, ensures uniqueness of the algorithm; on the other hand, it minimizes the load of the steering gear, because it minimizes the absolute difference between the current rudder angle and the control decision being generated.

The control algorithm can therefore be described in three steps:

(1) Determination of the future object states depending on the discretized control decisions

(2) Determining possible regions for an object state

(3) Determination of the control decision satisfying condition (8)

All the steps in the algorithm are sequential. The convergence of the algorithm is guaranteed by the feasibility of each step.

The idea behind the proposed control process is that at successive instants the generated control decisions will result in the object state getting to a region with a lower index. It follows that when at the instant zero the object state will be in the $k$ th region, such a control decision will be generated that at 1st instant the state object was in $k-1$ st region, until finally in $k$ th instant the object state should be found in the zero region and remain there, which is equivalent to bringing the ship's course to the set value (9).

The idea behind the proposed control process is as follows:

$$
\begin{aligned}
\underset{t=0}{O_{k}} \longrightarrow & \underset{t=1}{O_{k-1}} \longrightarrow O_{t=2} \longrightarrow \cdots \longrightarrow \underset{t=k-1}{O_{1}} \longrightarrow \underset{t=k}{O_{0}} \\
& \longrightarrow \underset{t=k+1}{O_{0}}
\end{aligned}
$$

The automatic control algorithm is required to guarantee the stability of the system. The relevant proof concerning the presented method of control is given in the next section.

2.3. The Control System Stability. The control system is called stable (in the Lyapunov sense) then and only then, if for every positive number $\varepsilon$ we can select such a number $\eta$ (generally dependent on $\varepsilon$ ) that a trajectory starting in a state $\mathbf{x}(0)$ lying inside the sphere with radius $\eta$ will remain inside the sphere with radius $\varepsilon$ for any instant $t$. In addition, if the condition occurs

$$
\lim _{t \rightarrow \infty} \mathbf{x}(t)=\mathbf{0}
$$

(i.e., for a time $t$ tending to infinity the control system returns to the state of equilibrium that had been disturbed), the control system is asymptotically stable. The stability of the control system near the point of equilibrium is called local stability, and the stability at any large initial conditions $\mathbf{x}(0)$ is called the global stability.

In order to show the global stability of the control system under consideration, let for any $\varepsilon$ meeting the condition $(2 \leq$ $i \leq k_{\text {max }}$, due to the definition of zero region, $d_{\text {max }}(1)$ is any small value, which can be considered approximately as zero)

$$
d_{\max }(i-1)<\varepsilon \leq d_{\max }(i),
$$


where

$d_{\max }(i)$ is the maximum distance (in the sense of Euclidean metric) of the boundary of $i$ th region from the point of equilibrium of the control system $(0 \leq i \leq$ $\left.k_{\max }\right)$.

$\eta$ will be selected as follows:

$$
\eta=d_{\min }(i-1),
$$

where

$d_{\min }(i)$ is the minimum distance (in the sense of Euclidean metric) of the boundary of $i$ th region from the point of equilibrium of the control system $(0 \leq i \leq$ $\left.k_{\max }\right)$.

Then the initial state of the control system, lying inside the sphere with radius $\eta$, must remain inside the sphere with radius $\varepsilon$, because in accordance with the control algorithm discussed in the previous section, it cannot leave the region it was previously found in, which completes the proof of stability in the Lyapunov sense of the proposed control system.

The operating principle of the control process described in the previous section was that in the subsequent instants such control decisions are generated that, when out of equilibrium, the system returns to the zero region, that is, the point of equilibrium, which fulfils condition (10), proving the asymptotic stability of the control system.

\section{Computing Experiments}

The experiments have been made in the MATLAB/Simulink environment. A de Wit-Oppe model [37] was used as an object (vessel), incorporating the dynamics of the steering gear [5]:

$$
\begin{aligned}
\dot{x}_{1} & =x_{5} \cos x_{3}-x_{6} \sin x_{3}, \\
\dot{x}_{2} & =x_{5} \sin x_{3}+x_{6} \cos x_{3}, \\
\dot{x}_{3} & =x_{4}, \\
\dot{x}_{4} & =-a_{1} x_{4}-a_{2} x_{4}{ }^{3}+a_{3} u, \\
\dot{x}_{5} & =-f x_{5}-W x_{4}{ }^{2}+S, \\
x_{6} & =-r_{1} x_{4}-r_{3} x_{4}{ }^{3}, \\
\dot{u} & =u_{z}-u, \\
\left|u_{z}\right| & \leq u_{\max }, \\
|\dot{u}| & \leq \dot{u}_{\max },
\end{aligned}
$$

where

$\left(x_{1}, x_{2}\right)=(x, y)$ is Cartesian coordinates (ship's position),

$x_{3}=\psi$ is deviation from the course,

$x_{4}=r$ is angular velocity, $x_{5}$ is longitudinal speed,

$x_{6}$ is lateral speed,

$u=\delta$ is rudder angle,

$u_{z}=\delta_{z}$ is preset rudder angle,

$\delta_{\max }$ is maximum rudder angle,

$\dot{\delta}_{\max }$ is maximum rate of turn of the rudder,

$S$ is propeller thrust,

$a_{1}, a_{2}, a_{3}, f, W, r_{1}$, and $r_{3}$ are coefficients determined from model tests (different for different types of vessels).

The ship movement parameters assumed here are those of a ship of mariner class, such as the m.s. Compass Island [37]: $a_{1}=0,018[1 / \mathrm{s}], a_{2}=37,2\left[\mathrm{~s} / \mathrm{rad}^{2}\right], a_{3}=0,001\left[1 / \mathrm{s}^{2}\right]$, $f=0,014[1 / \mathrm{s}], W=124\left[\mathrm{~m} / \mathrm{rad}^{2}\right], S=0,11\left[\mathrm{~m} / \mathrm{s}^{2}\right], r_{1}=$ $-69,5[\mathrm{~m} / \mathrm{rad}]$, and $r_{3}=0\left[\mathrm{~m} \cdot \mathrm{s}^{2} / \mathrm{rad}^{3}\right]$. The selected ship has the following characteristics: gross registered tons $9214[\mathrm{t}]$, 13498 DWT $[\mathrm{t}]$, single screw, length $172[\mathrm{~m}]$, maximum draft $8[\mathrm{~m}]$, maximum speed $20[\mathrm{kn}]$, maximum (minimum) angular velocity $r_{\max }=0,0191[\mathrm{rad} / \mathrm{s}]\left(r_{\min }=-0,0191[\mathrm{rad} / \mathrm{s}]\right)$, maximum (minimum) rudder angle $\delta_{\max }=0,6[\mathrm{rad}]\left(\delta_{\min }=\right.$ $-0,6[\mathrm{rad}])$, and maximum (minimum) rate of turn of the rudder $\dot{\delta}_{\max }=0,066[\mathrm{rad} / \mathrm{s}]\left(\dot{\delta}_{\min }=-0,066[\mathrm{rad} / \mathrm{s}]\right)$.

In order to take account of disturbances, simulations included a signal characteristic of wind-induced sea waves [5].

There were two computing experiments. Each of them consisted of simulation series comparing the operation of the proposed system to the control performed by an LQR controller. For comparison, an LQR regulator was chosen, as it is an optimal regulator for a linear stationary object. A Nomoto model was used as a ship's model for the synthesis of the $\mathrm{LQR}$ regulator [5]:

$$
\begin{aligned}
& \dot{x}_{3}=x_{4}, \\
& \dot{x}_{4}=-a_{1} x_{4}+a_{3} u .
\end{aligned}
$$

As a quality criterion, this functional was taken:

$$
J=\int_{0}^{t}\left(\psi^{2}+\lambda \delta^{2}\right) d t
$$

where

$\lambda$ is a coefficient greater than zero, which is interpreted as a compromise between the deviation from the course (angle of yaw) and the rudder angle (steering gear load), arbitrarily adopted here as 1 .

Then the control law for LQR can be described by this formula:

$$
\delta=k_{\psi} \psi+k_{r} r
$$

where

$$
\begin{aligned}
& k_{\psi}=-\frac{1}{\sqrt{\lambda}}, \\
& k_{r}=\frac{1}{a_{3}}\left(a_{1}-\sqrt{a_{1}^{2}+\frac{2 a_{3}}{\sqrt{\lambda}}}\right) .
\end{aligned}
$$



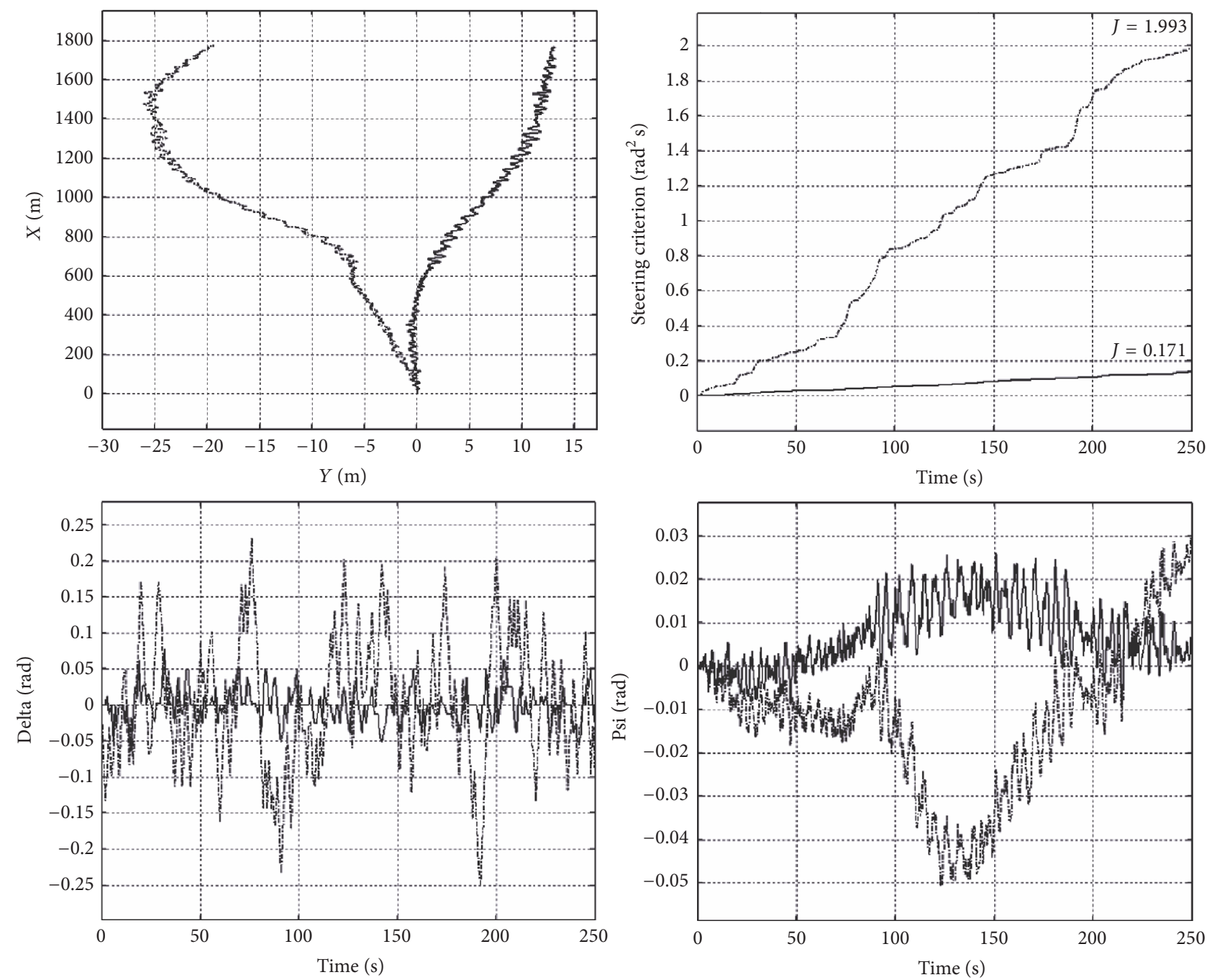

FIGURE 3: Comparison of the proposed system operation (continuous line) and an LQR controller (dashed line), experiment one.

The first computing experiment was aimed to compare the values of quality control indicator (15) measured (for a time span of 0-250 seconds) for different types of waves, for a ship with the course set on zero radians. Figure 3 presents an example simulation result for sea state 4 . The charts, respectively, depict the trajectory of ship movement, the indicator of quality control, the rudder angle, and the deviation from the course in the simulation. The values of quality control indicator should be interpreted accordingly as $0.1993 \%$ and $0.0171 \%$ decrease in ship's operational speed caused by additional resistance associated with disturbances. By using the proposed method, the decrease in speed was much smaller. These results clearly confirm high quality of the proposed system operation.

In all the cases examined, the quality indicator value for a ship controlled by the proposed system was lower than in the system using an LQR controller (even if the latter is optimal for linear stationary object). The developed method allowed reducing the decrease in ship's operational speed by $0.1 \%-0.5 \%$, depending on wave height. It was also observed that the control quality was improving, compared to the LQR controller, with increasing wave height.

The other computing experiment was to examine how much time it takes for the object to reach the zero region (i.e., vessel is brought on a new course), if at instant zero the course is altered from 0 radians to a new course other than zero. In other words, the test included the time of course stabilization (control time) and overshoot, in a situation of an abrupt change of the setpoint. Figure 4 presents an example simulation result obtained during the tests. The charts illustrate the trajectory of ship movement, the indicator of quality control, the rudder angle, and the deviation from the course in the simulation. In this situation, the course was changed from 0 to $\pi / 4$ radian. We can observe a shorter transitional period in the proposed control method. These results, like in the first experiment, confirm high quality of the proposed system.

In all the cases examined, the time to reach the zero region by a ship controlled by the proposed system was less than in case of the control involving an LQR controller. Owing to the developed method, course stabilization time was shortened 

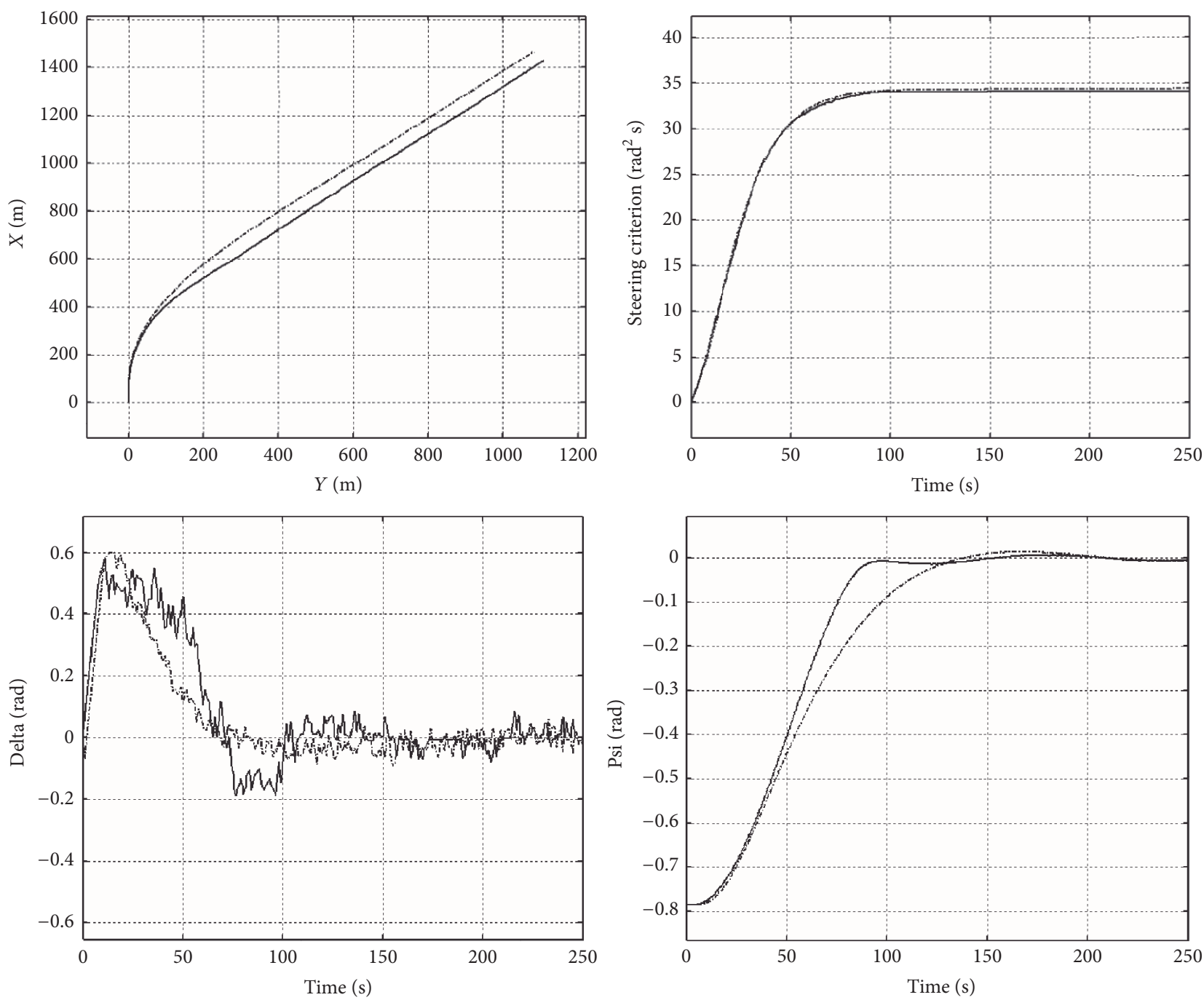

FIGURE 4: Comparison of the proposed system operation (continuous line) and an LQR controller (dashed line), experiment two.

approximately from $5 \%$ to $40 \%$, depending on the value of the newly defined course.

The computing experiments presented in this section clearly confirm high quality of control (ship course stabilization) of the proposed expert system. This refers to the minimization of control criterion value (15), control time, and the magnitude of overshoot at a step alteration of the ship's course. In all the cases examined, the control quality of the proposed system was higher than that of an LQR controller.

\section{Conclusions}

One of the important tasks in maritime transport, which has an impact on the reduction of transport costs and enhancement of navigation safety, is the problem of automatic ship's course stabilization. However, the main difficulties that appear in the design of efficient navigational devices are problems with developing effective algorithms implementing desired functionalities. Designing solutions that will be sufficiently effective and based on few simplifications leads to complex systems that are hard to be analyzed in a traditional manner. Hence, it seems necessary to apply computational mathematics for solving problems of marine navigation, or, in a broader perspective, maritime transport. Computational mathematics is understood here as a combination of traditional mathematical tools, characterized by strict reasoning (strict evidence) and artificial intelligence methods, additionally supported by technical capabilities of modern computers [38]. The expert system discussed herein, tasked to automatically stabilize ship's course, fits well into that area of research. By using the data on the system hydrodynamics and the original inference engine, we could bypass difficulties that occur in developing typical control algorithms for complex and nonlinear model affected by strong external disturbances (wind, waves), while providing high quality of control. This was confirmed by computing experiments.

The proposed system will be implemented in the executive module of the navigational decision support system NAVDEC [39-41]. The executive module combined with other modules (e.g., module of data fusion [42-45] and 
prediction module $[46,47])$ is part of the navigational decision support system in the process of safe ship conduct (invention [48]).

The developed system has a function of automatic ship's course stabilization. However, the methodology can be easily extended and used for solving problems of automatic ship steering along a preset trajectory. It seems that in the context of the proposed approach it is possible, for further considerations, to extend tasks and include other objects of control. Hence, the proposed methodology represents a new branch of intelligent control systems.

\section{Conflicts of Interest}

The author declares no conflicts of interest.

\section{Acknowledgments}

This research outcome has been achieved under a grant financed from a subsidy of the Ministry of Science and Higher Education in Poland for statutory activities (no. 1/S/ITM/16).

\section{References}

[1] Review of maritime transport 2016; Report by the United Nations Conference on Trade and Development: Geneva, Switzerland, 2016.

[2] S. Guze, L. Smolarek, and A. Weintrit, "The area-dynamic approach to the assessment of the risks of ship collision in the restricted water," Scientific Journals of the Maritime University of Szczecin, vol. 117, pp. 88-93, 2016.

[3] N. Minorsky, "Directional stability of automatically steered bodies," Journal of American Society of Naval Engineers, vol. 34, pp. 280-309, 1922.

[4] E. Sperry, "Automatic steering," in Proceedings of the 30th Meeting of the Society of Naval Architects and Marine Engineers, pp. 53-61, 1922.

[5] T. I. Fossen, Guidance and Control of Ocean Vehicles, John Wiley and Sons, New York, NY, USA, 1994.

[6] T. I. Fossen, Handbook of Marine Craft Hydrodynamics and Motion Control, John Wiley Sons, New York, NY, USA, 2011.

[7] T. Perez, Ship Motion Control, Springer-Verlag, London, UK, 2005.

[8] J. Van Amerongen, "Adaptive steering of ships-a model reference approach," Automatica, vol. 20, no. 1, pp. 3-14, 1984.

[9] J. Van Amerongen and A. J. Udink Ten Cate, "Model reference adaptive autopilots for ships," Automatica, vol. 11, no. 5, pp. 441449, 1975.

[10] K. J. Astrom and C. G. Kallström, "Identification of ship steering dynamics," Automatica, vol. 12, no. 1, pp. 9-22, 1976.

[11] T. Holzhuter and H. Strauch, "A commercial adaptive autopilot for ships," in Proceedings of the 10th IFAC World Congress, pp. 226-230, 1987.

[12] C. G. Kallstrom and K. J. Astrom, "Experiences of system identification applied to ship steering," Automatica, vol. 17, no. 1, pp. 187-198, 1981.

[13] C. G. Kallstrom, K. J. Astrom, N. E. Thorell, J. Eriksson, and L. Sten, "Adaptive autopilots for tankers," Automatica, vol. 15, no. 3, pp. 241-254, 1979.
[14] L. Morawski and J. Pomirski, "Design of the robust PID coursekeeping control system for ship," Polish Maritime Reesearch, pp. 20-23, 2002.

[15] K. Ohtsu, M. Horigome, and G. Kitagawa, "A new ship's auto pilot design through a stochastic model," Automatica, vol. 15, no. 3, pp. 255-268, 1979.

[16] P. Borkowski, "Ship course stabilization by feedback linearization with adaptive object model," Polish Maritime Research, vol. 21, no. 1, pp. 14-19, 2014.

[17] T. I. Fossen and M. J. Paulsen, "Adaptive feedback linearization applied to steering of ships," in Proceedings of the The First IEEE Conference on Control Applications, pp. 1088-1093, 1992.

[18] L. Morawski, J. Pomirski, and H. Siguerdidjane, "Nonlinear ship autopilot," in Proceedings of the 2nd International Congress of Marine Technological Innovations and Research, pp. 559-566, 2000.

[19] X. Pend, S. Jia, and Z. Hu, "Nonlinear H-infinity inverse optimal output feedback control for ship course," Control Theory and Applications, vol. 31, pp. 215-222, 2014.

[20] L. P. Perera and C. Guedes Soares, "Pre-filtered sliding mode control for nonlinear ship steering associated with disturbances," Ocean Engineering, vol. 51, pp. 49-62, 2012.

[21] X. Sun and W. Chen, "Global generalised exponential/finitetime control for course-keeping of ships," International Journal of Control, vol. 89, no. 6, pp. 1169-1179, 2016.

[22] M. Tomera, "Nonlinear controller design of a ship autopilot," International Journal of Applied Mathematics and Computer Science, vol. 20, no. 2, pp. 271-280, 2010.

[23] C. Y. Tzeng, G. C. Goodwin, and S. Crisafulli, "Feedback linearization design of a ship steering autopilot with saturating and slew rate limiting actuator," International Journal of Adaptive Control and Signal Processing, vol. 13, no. 1, pp. 23-30, 1999.

[24] A. Witkowska, M. Tomera, and R. Śmierzchalski, "A backstepping approach to ship course control," International Journal of Applied Mathematics and Computer Science, vol. 17, no. 1, pp. 7385, 2007.

[25] Z. Zwierzewicz, "On the ship course-keeping control system design by using robust feedback linearization," Polish Maritime Research, vol. 20, no. 1, pp. 70-76, 2013.

[26] P. Borkowski and Z. Zwierzewicz, "Ship course-keeping algorithm based on knowledge base," Intelligent Automation and Soft Computing, vol. 17, no. 2, pp. 149-163, 2011.

[27] M. Le, S. Nguyen, and L. Nguyen, "Study on a new and effective fuzzy PID ship autopilot," Artificial Life and Robotics, vol. 8, no. 2, pp. 197-201, 2004.

[28] S. Liu, Y.-C. Wang, and H.-X. Fu, "Variable universe fuzzy-least squares support-vector-machine compound control for ship course-keeping," Kongzhi Lilun Yu Yingyong/Control Theory and Applications, vol. 28, no. 4, pp. 485-490, 2011.

[29] E. W. McGookin, D. J. Murray-Smith, Y. Li, and T. I. Fossen, "The optimization of a tanker autopilot control system using genetic algorithms," Transactions of the Institute of Measurement \& Control, vol. 22, no. 2, pp. 141-178, 2000.

[30] L. Morawski and A. Rak, "Neural network application to course control of large tanker," in Proceedings of the 8th IEEE International Conference of Methods and Models in Automation and Robotics, pp. 847-850, Poland, 2002.

[31] V. Nicolau, "Neuro-fuzzy system for intelligent course control of underactuated conventional ships," in Proceedings of the 2nd IEEE International Workshop on Soft Computing Applications, SOFA 2007, pp. 95-101, Romania, August 2007. 
[32] J. Ren and X. Zhang, "Ship course-keeping adaptive Fuzzy controller design using command filtering with minimal parametrization," in Proceedings of the 2013 25th Chinese Control and Decision Conference, CCDC 2013, pp. 243-247, China, May 2013.

[33] M. A. Unar and D. J. Murray-Smith, "Automatic steering of ships using neural networks," International Journal of Adaptive Control and Signal Processing, vol. 13, no. 4, pp. 203-218, 1999.

[34] H. Xu, W. Li, Y. Yu, and Y. Liu, "A novel adaptive neural control scheme for uncertain ship course-keeping system," Sensors Transducers, vol. 178, pp. 282-285, 2014.

[35] Y. Han, H. Xiao, W. Pan, C. Wang, and F. Zhou, "A fuzzy sliding mode controller and its application on ship course control," in Proceedings of the 2010 7th International Conference on Fuzzy Systems and Knowledge Discovery, FSKD 2010, pp. 635-638, China, August 2010.

[36] A. Zirilli, G. N. Roberts, A. Tiano, and R. Sutton, "Adaptive steering of a containership based on neural networks," International Journal of Adaptive Control and Signal Processing, vol. 14, no. 8, pp. 849-873, 2000.

[37] C. de Wit and J. Oppe, "Optimal Collision Avoidance in Unconfined Waters," Journal of the Institute of Navigation, vol. 26, no. 4, pp. 296-303, 1979.

[38] P. Borkowski, "Computational mathematics in marine navigation," Scientific Journals Maritime University of Szczecin, vol. 21, pp. 20-27, 2010.

[39] P. Borkowski, "Presentation algorithm of possible collision solutions in a navigational decision support system," Scientific Journals Maritime University of Szczecin, vol. 38, pp. 20-26, 2014.

[40] Z. Pietrzykowski, P. Borkowski, and P. Wołejsza, "NAVDEC navigational decision support system on a sea going vessel," Scientific Journals Maritime University of Szczecin, vol. 30, pp. 102-108, 2012.

[41] Z. Pietrzykowski, P. Wołejsza, and P. Borkowski, "Decision Support in Collision Situations at Sea," Journal of Navigation, vol. 70, no. 3, pp. 447-464, 2017.

[42] P. Borkowski, J. Magaj, and M. Maka, "Positioning based on the multi-sensor Kalman filter," Scientific Journals Maritime University of Szczecin, vol. 13, pp. 5-9, 2008.

[43] W. Kazimierski, "Proposal of neural approach to maritime radar and automatic identification system tracks association," IET Radar, Sonar \& Navigation, vol. 11, no. 5, pp. 729-735, 2017.

[44] W. Kazimierski and A. Stateczny, "Radar and Automatic Identification System Track Fusion in an Electronic Chart Display and Information System," Journal of Navigation, vol. 68, no. 6, pp. 1141-1154, 2015.

[45] G. Zaniewicz, W. Kazimierski, and I. Bodus-Olkowska, "Integration of Spatial Data from External Sensors in the Mobile Navigation System for Inland Shipping," in Proceedings of the 2016 Baltic Geodetic Congress (Geomatics), BGC Geomatics 2016, pp. 165-170, Poland, June 2016.

[46] P. Borkowski, "The Ship Movement Trajectory Prediction Algorithm Using Navigational Data Fusion," Sensors, vol. 17, no. 6, p. 1432, 2017.

[47] M. Kijewska, "Route prediction for a person in water drifting in chosen basins using graph theory," Scientific Journals Maritime University of Szczecin, vol. 50, pp. 45-51, 2017.

[48] Z. Pietrzykowski and P. Borkowski, "A method and system of navigational decision support in the process of safe vessel navigation," in Proceedings of the International patent application under PCT procedure, pp. 08-11, 2010. 

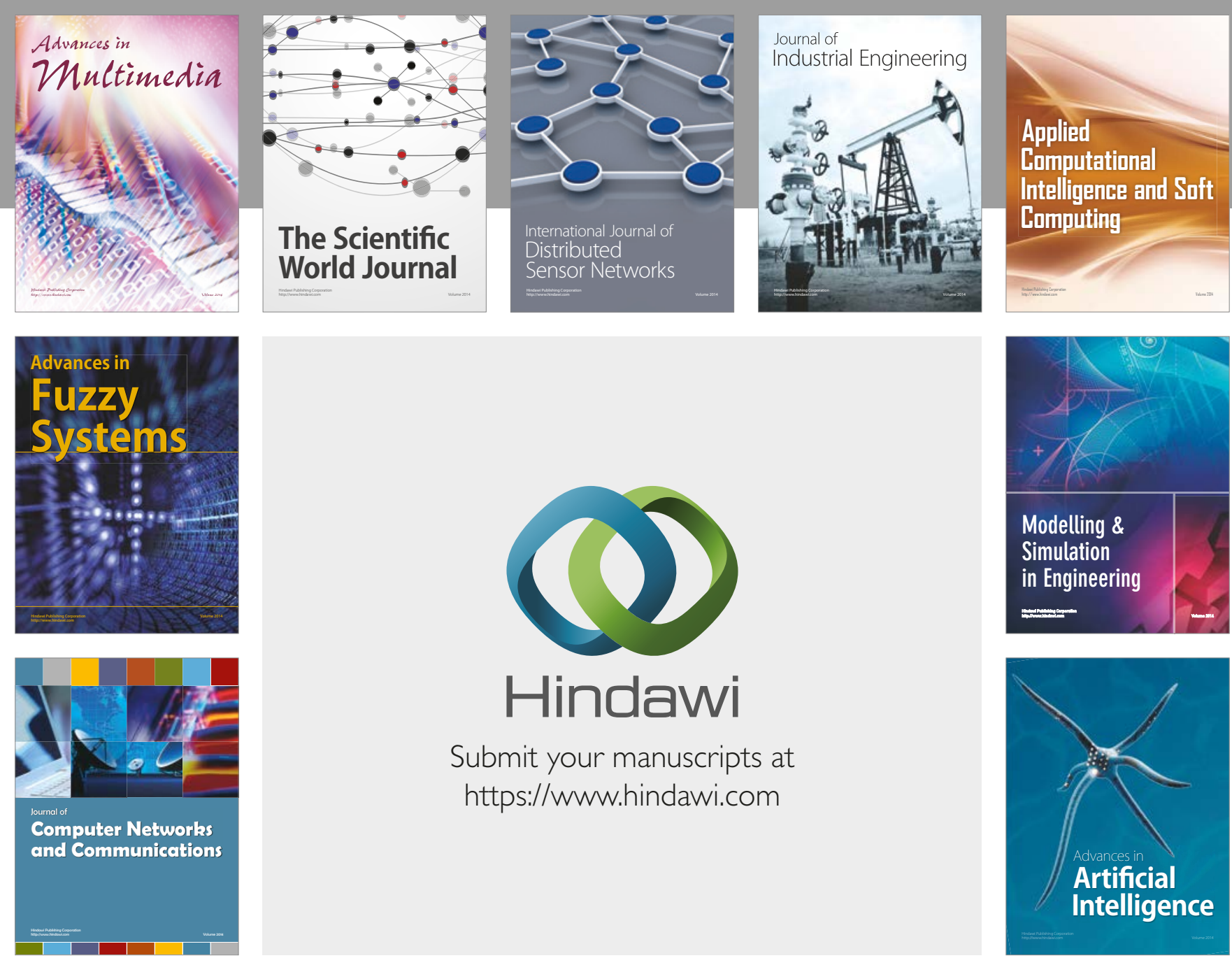

\section{Hindawi}

Submit your manuscripts at

https://www.hindawi.com
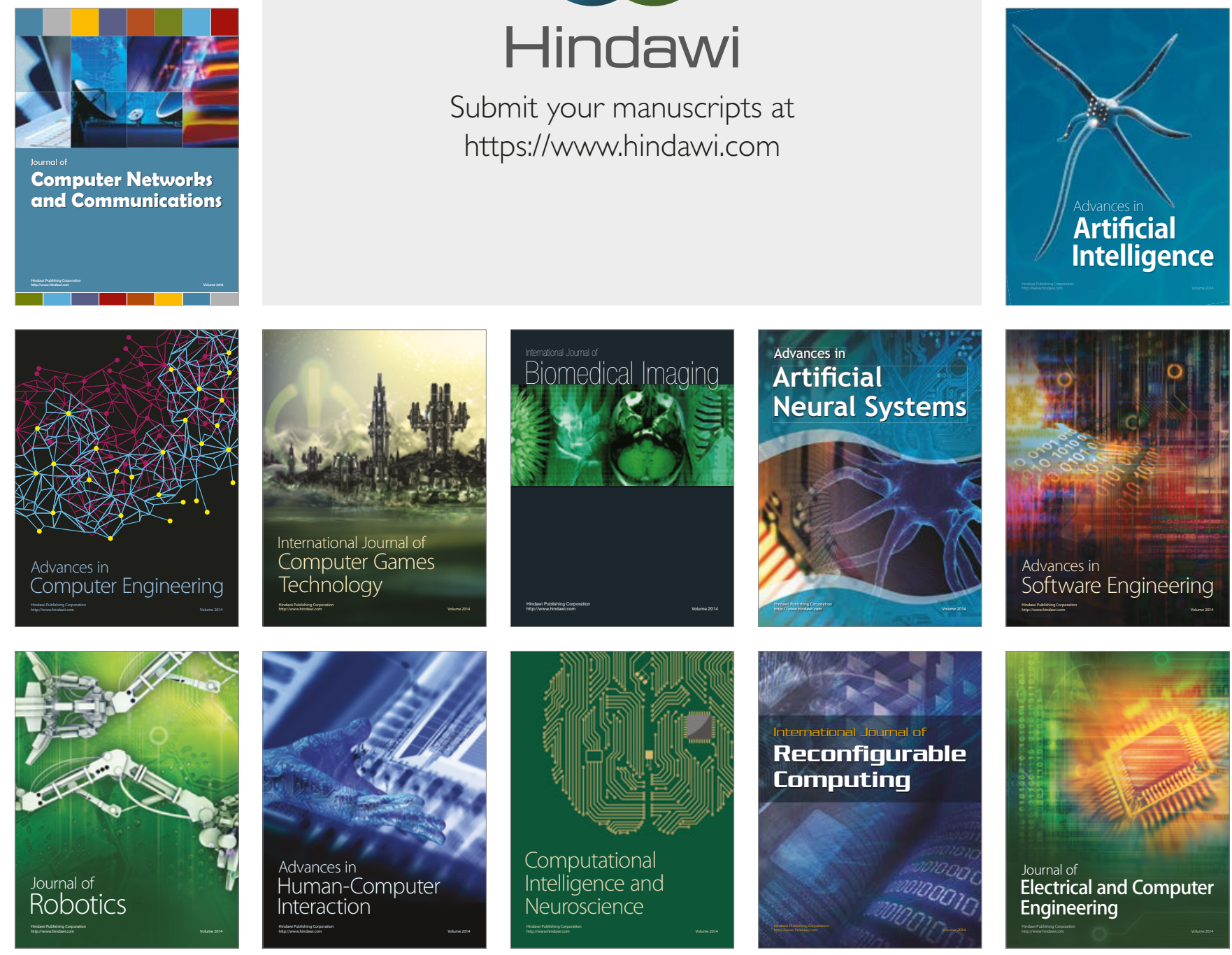